\title{
ALTERAÇÕES ELETROQUÍMICAS E DINÂMICA DE NUTRIENTES NA SOLUÇÃO DO SOLO EM ARROZ IRRIGADO COM LIXIVIADO INDUSTRIAL TRATADO
}

\author{
Filipe Selau Carlos ${ }^{(1)}$, Andrei José Marafon(1), Robson Andreazza( ${ }^{(2)}$, Ibanor Anghinoni ${ }^{(1)}$, \\ Marino José Tedesco(1) e Flávio Anastácio de Oliveira Camargo ${ }^{(1) *}$
}

(1) Universidade Federal do Rio Grande do Sul, Departamento de Solos, Porto Alegre, Rio Grande do Sul, Brasil.

(2) Universidade Federal de Pelotas, Centro de Engenharias, Pelotas, Rio Grande do Sul, Brasil.

* Autor correspondente.

E-mail: fcamargo@ufrgs.br

\section{RESUMO}

A utilização de efluentes industriais tratados na irrigação do arroz por alagamento pode provocar alterações eletroquímicas e aumentar o teor de nutrientes na solução do solo. Para testar essa hipótese, este trabalho teve por objetivo avaliar a dinâmica dos atributos químicos e eletroquímicos da solução do solo sob cultivo de arroz irrigado com lixiviado industrial tratado, contendo $820 \mathrm{mg} \mathrm{L}^{-1}$ de $\mathrm{Na}$. O experimento foi conduzido em casa de vegetação, utilizando-se como unidades experimentais vasos preenchidos com $20 \mathrm{~kg}$ de solo, em delineamento experimental em blocos casualizados, com três repetições. Os tratamentos foram: controle (irrigação com água destilada) e quatro proporções do lixiviado $(25,50,75$ e $100 \%$ ). As coletas de solução do solo foram feitas semanalmente a partir do quarto dia após o início do alagamento (DAA) até 84 DAA. A solução do solo foi amostrada na profundidade de 10 cm e analisada para os principais nutrientes e o $\mathrm{Na}$, bem como para a demanda bioquímica de oxigênio $\left(\mathrm{DBO}_{5}\right)$, relação de adsorção de sódio (RAS), condutividade elétrica (CE) e potencial redox $\left(\mathrm{E}_{\mathrm{H}}\right)$. A irrigação com o lixiviado aumentou os teores de $\mathrm{K}, \mathrm{Ca}, \mathrm{Mg}, \mathrm{S}, \mathrm{P}, \mathrm{N}-\mathrm{NH}_{4}^{+}, \mathrm{N}-\mathrm{NO}_{3}^{-} \mathrm{e}$ $\mathrm{Na}$, assim como os valores de RAS e CE, para valores considerados prejudiciais para as plantas. Foi observada diminuição do potencial redox na solução do solo pela irrigação com lixiviado industrial tratado. Os teores de $\mathrm{DBO}_{5}$ e o N-NH${ }_{4}^{+}$diminuíram com o tempo de alagamento. Em proporções menores que $25 \%$, o lixiviado industrial tratado pode aumentar os teores de nutrientes em solução sem causar interferência do Na para as plantas.

Palavras-chave: águas residuárias, Oriza sativa, potencial redox, nitrogênio, $\mathrm{DBO}_{5}$. 


\title{
ABSTRACT: ELECTROCHEMICAL CHANGES AND NUTRIENT DYNAMICS IN THE SOLUTION OF SOIL WITH RICE IRRIGATED WITH TREATED INDUSTRIAL LEACHATE
}

\begin{abstract}
The use of treated industrial wastewater in flood irrigation of rice may cause electrochemical changes and increase the nutrient content in the soil solution. To test this hypothesis, the aim of the present study was to evaluate the dynamics of the chemical and electrochemical properties of the soil solution under flooded rice irrigated with treated industrial leachate containing $820 \mathrm{mg} \mathrm{L}^{-1}$ of $\mathrm{Na}$. The experiment was conducted in a greenhouse, using pots filled with $20 \mathrm{~kg}$ of soil in a randomized block design with three replications. The treatments were control (irrigation with distilled water) and four ratios of the leachate (25, 50, 75, and $100 \%$ ). Samples of soil solution were taken weekly from the fourth day after the start of flooding (DAF) until 84 DAF, The soil solution was sampled by means of $50 \mathrm{~mm}$ diameter PVC collectors at $10 \mathrm{~cm}$ depth, and analyzed for key nutrients and $\mathrm{Na}$, as well as for biochemical oxygen demand (BOD), sodium adsorption ratio (SAR), electrical conductivity $(E C)$, and redox potential $\left(E_{H}\right)$. Irrigation with leachate increased the levels of $\mathrm{Ca}, \mathrm{Mg}, \mathrm{S}, \mathrm{P}, \mathrm{NH}_{4}^{+}-\mathrm{N}$, $\mathrm{NO}_{3}^{-} \mathrm{N}$ and $\mathrm{Na}$, as well as the values of the $\mathrm{SAR}$ and the value of $\mathrm{EC}$, to levels considered harmful to plants. $\mathrm{A}$ decrease in $E_{H}$ was observed in the soil solution through irrigation with treated industrial leachate. The levels of $\mathrm{BOD}_{5}$ and $\mathrm{NH}_{4}^{+}-\mathrm{N}$ decreased with time of flooding. At proportions of less than $25 \%$, the treated industrial leachate can increase the levels of nutrients in the soil solution without Na reaching critical levels for plants.
\end{abstract}

Keywords: wastewater, Oryza sativa, redox potential, nitrogen, $B O D_{5}$.

\section{INTRODUÇÃO}

Efluentes industriais tratados inadequadamente estão entre os principais responsáveis pela contaminação hídrica do delta do Jacuí, onde deságuam três dos 10 rios mais poluídos do Brasil (IBGE, 2010). Observa-se grande contribuição de fontes pontuais e difusas de poluição, com destaque para as águas residuárias que possuem alto teor de $\mathrm{C}$ orgânico dissolvido e altos teores de $\mathrm{N}$, sais e outros compostos (Fonseca et al., 2007). Nessas condições, verifica-se a diminuição nos teores de oxigênio dissolvido nas águas e os problemas decorrentes como morte de peixes e outros desequilíbrios nos mananciais hídricos (Feigin et al., 1991; Al-Omari e Fayyad, 2003).

Alternativas para a disposição de águas residuárias são necessárias para a redução dos desequilíbrios em corpos d'água (Hamilton et al., 2007). O uso agrícola dos efluentes industriais tratados tem grande potencial (Feigin et al., 1991), pois os nutrientes presentes nessas águas podem favorecer o desenvolvimento de plantas como o arroz. Essa cultura é um cereal de importância econômica, produzido em solos de várzea sob irrigação por alagamento, predominantemente nos Estados do Rio Grande do Sul e de Santa Catarina (Sosbai, 2012). A irrigação com águas residuárias aumenta a absorção de N, P, K e outros elementos pelas plantas com aumento no rendimento das culturas (Fonseca et al., 2007; Lourenzi et al., 2013). Em contrapartida, alguns sais e elementos, como o Na, encontram-se em altas concentrações em efluentes tratados e podem atuar como agentes dispersantes da estrutura do solo, comprometendo o desenvolvimento de plantas (Leal et al., 2009; Blum et al., 2012). Devese considerar, ainda, que os ambientes de várzeas encontram-se próximos dos rios e do lençol freático e exigem monitoramento constante dos potenciais contaminantes aplicados com as águas residuárias.

Além dos possíveis problemas da disposição de águas residuárias para a irrigação do arroz, o alagamento também altera características eletroquímicas da solução do solo. Com a inundação ocorre rápido consumo de oxigênio e uma transição da população microbiana aeróbia para anaeróbia com redução dos compostos oxidados, utilizados como aceptores de elétrons, alterando a dinâmica dos íons em solução (Ponnamperuma, 1972; Sousa et al., 2002). Elementos como sais, N em formas minerais e carbono orgânico dissolvido (COD) encontram-se em altas concentrações em efluentes tratados (Fonseca et al., 2005) e este último altera a velocidade das reações redox do solo (Camargo et al., 1993). Os altos teores de $\mathrm{Na}, \mathrm{Ca}, \mathrm{Mg}$ e K encontrados nos efluentes também modificam a composição da solução e podem influenciar o desenvolvimento de plantas de arroz. Com base nessas constatações, este trabalho teve por objetivo avaliar a dinâmica dos atributos químicos e eletroquímicos da solução do solo sob cultivo de arroz irrigado com lixiviado industrial tratado, contendo $820 \mathrm{mg} \mathrm{L}^{-1}$ de $\mathrm{Na}$.

\section{MATERIAL E MÉTODOS}

O experimento foi conduzido em casa de vegetação nas instalações do Departamento de Solos da Faculdade de Agronomia, da Universidade 
Federal do Rio Grande do Sul, nas coordenadas geográficas $30^{\circ} 01^{\prime} 58^{\prime \prime} \mathrm{Se}$ e $51^{\circ} 13^{\prime} 48^{\prime \prime} \mathrm{O}$. As unidades experimentais foram constituídas por vasos com $20 \mathrm{~L}$ de capacidade e preenchidos com $20 \mathrm{~kg}$ de solo. Os tratamentos foram delineados em blocos casualizados, com três repetições e consistiram de composições de irrigação com as seguintes proporções: controle (100 \% água destilada e $0 \%$ lixiviado), T25 (75 \% água destilada e $25 \%$ lixiviado), T50 (50\% água destilada e $50 \%$ lixiviado), T75 (25\% água destilada e $75 \%$ lixiviado) e T100 (0\% água destilada e $100 \%$ lixiviado). O solo utilizado foi classificado como Gleissolo Háplico (Embrapa, 2006), que após coletado foi analisado química e fisicamente (Tedesco et al., 1995). A caracterização química do solo previamente à instalação do experimento é apresentada no quadro 1.

O lixiviado industrial tratado (Quadro 2) foi coletado na central de recebimento de resíduos industriais UTRESA, localizada em Estância Velha, RS. O lixiviado é uma água residuária originada da decomposição dos resíduos sólidos, principalmente aparas de couro, contidas em células de aterro. Após geração, os lixiviados são bombeados para a estação de tratamento de efluentes (ETE), onde recebem tratamentos primário e secundário. $\mathrm{Na}$ etapa subsequente, são armazenados em lagoa de estabilização de onde foi coletado para aplicação no experimento.

O solo coletado foi corrigido com a adição de dose equivalente a 1,3 t ha ${ }^{-1}$ de corretivo $\left(\mathrm{CaCO}_{3}+\mathrm{MgCO}_{3}\right.$ na proporção $3: 1$ ) para elevar o $\mathrm{pH}$ a 5,5 , conforme recomendações da CQFS-RS/SC (2004). Após

Quadro 1. Atributos químicos do Gleissolo Háplico Amarelo antes da instalação do experimento

\begin{tabular}{|c|c|}
\hline Atributo & Valor \\
\hline Argila $\left(\mathrm{kg} \mathrm{kg}^{-1}\right)$ & 0,170 \\
\hline Matéria orgânica (\%) & 2,3 \\
\hline $\mathrm{pH}\left(\mathrm{H}_{2} \mathrm{O}\right)(1: 1)$ & 4,7 \\
\hline $\mathrm{H}+\mathrm{Al}\left(\mathrm{cmol}_{\mathrm{c}} \mathrm{dm}^{-3}\right)$ & 4,4 \\
\hline Índice SMP & 6,0 \\
\hline $\mathrm{Ca}^{2+}\left(\mathrm{cmol}_{\mathrm{c}} \mathrm{dm}^{-3}\right)$ & 1,3 \\
\hline $\mathrm{Mg}^{2+}\left(\mathrm{cmol}_{\mathrm{c}} \mathrm{dm}^{-3}\right)$ & 0,3 \\
\hline $\mathrm{CTC}_{\mathrm{pH} 7,0}\left(\mathrm{cmol}_{\mathrm{c}} \mathrm{dm}^{-3}\right)$ & 6,1 \\
\hline Saturação por bases (\%) & 28 \\
\hline $\mathrm{Al}^{3+}\left(\mathrm{cmol}_{\mathrm{c}} \mathrm{dm}^{-3}\right)$ & 0,90 \\
\hline Saturação por alumínio (\%) & 34 \\
\hline $\mathrm{K}^{+}\left(\mathrm{cmol}_{\mathrm{c}} \mathrm{dm}^{-3}\right)$ & 0,12 \\
\hline $\mathrm{P}\left(\mathrm{mg} \mathrm{dm}{ }^{-3}\right)$ & 9,10 \\
\hline $\mathrm{S}\left(\mathrm{mg} \mathrm{dm}^{-3}\right)$ & 17 \\
\hline $\mathrm{Zn}\left(\mathrm{mg} \mathrm{dm}^{-3}\right)$ & 3,7 \\
\hline $\mathrm{Cu}\left(\mathrm{mg} \mathrm{dm}{ }^{-3}\right)$ & 0,7 \\
\hline $\operatorname{Mn}\left(\mathrm{mg} \mathrm{dm}^{-3}\right)$ & 0,2 \\
\hline
\end{tabular}

30 dias, foi realizada a adubação na base, com a aplicação de $50 \mathrm{~kg} \mathrm{ha}^{-1}$ de $\mathrm{P}_{2} \mathrm{O}_{5}$ (superfosfato triplo) e $100 \mathrm{~kg} \mathrm{ha}^{-1}$ de $\mathrm{K}_{2} \mathrm{O}(\mathrm{KCl})$, e, quando a planta estava em estádio V4 (quatro folhas completamente expandidas), foram aplicados $2 / 3$ da dose recomendada (150 kg de $\mathrm{N} \mathrm{ha}^{-1}$, na forma de ureia) e 1/3 em V6-V7 (Sosbai, 2012), em todos os tratamentos. Foram semeadas 10 sementes de arroz, cultivar IRGA 424, e após a completa germinação as plântulas foram desbastadas deixando as três mais vigorosas por vaso. As plântulas foram irrigadas com água destilada, à capacidade de campo, até o estádio V3-V4. Em seguida, foram aplicados os tratamentos, concomitantemente ao início da aplicação da lâmina de irrigação, até o final do experimento. Aos 18 e 46 dias após o alagamento (DAA), foi aplicada quantidade equivalente a $100 \mathrm{~mm}$ de chuva (7 L/vaso) para simular a retirada de sal que ocorreria em uma situação de campo. O mesmo volume de solução do solo foi retirado por um sifão na parte inferior do vaso, fazendo com que o volume de água aplicado $(7 \mathrm{~L})$ passasse pela massa de solo, lixiviando os sais. A irrigação dos vasos sempre manteve uma lâmina de $10 \mathrm{~cm}$. Na figura 1, são apresentados os volumes de irrigação em cada tratamento. A diferença de volume entre os tratamentos se deve às diferenças no desenvolvimento das plantas. As quantidades de fertilizantes adicionadas pela irrigação foram calculadas pelos volumes de irrigação de cada tratamento e pelo teor médio dos elementos no lixiviado industrial tratado. Posteriormente, estimaram-se as doses considerando a massa de 2.000.000 kg ha-1 de solo.

Quadro 2. Características do lixiviado industrial tratado (LIT) e padrões para lançamento em corpos hídricos

\begin{tabular}{lcc}
\hline Atributo & Valor & Padrão de lançamento \\
\hline $\mathrm{pH}$ & 7,9 & $6,0-9,0$ \\
$\mathrm{~N}$ total $\left(\mathrm{mg} \mathrm{L}^{-1}\right)$ & 217 & 20 \\
$\mathrm{Ca}\left(\mathrm{mg} \mathrm{L}^{-1}\right)$ & 37 & - \\
$\mathrm{Mg}\left(\mathrm{mg} \mathrm{L}^{-1}\right)$ & 47 & - \\
$\mathrm{K}\left(\mathrm{mg} \mathrm{L}^{-1}\right)$ & 120 & - \\
$\mathrm{Na}$ total $\left(\mathrm{mg} \mathrm{L}^{-1}\right)$ & 820 & - \\
$\mathrm{S}\left(\mathrm{mg} \mathrm{L}^{-1}\right)$ & 21 & - \\
$\mathrm{P}$ total $\left(\mathrm{mg} \mathrm{L}^{-1}\right)^{(2)}$ & 1,0 & 2,9 \\
$\mathrm{CE}\left(\mu \mathrm{cm}^{-1}\right)$ & 6.810 & - \\
$\mathrm{DQO}\left(\mathrm{mg} \mathrm{L}^{-1}\right)$ & 280 & 330 \\
$\mathrm{DBO}_{5}\left(\mathrm{mg} \mathrm{L}^{-1}\right)$ & 100 & 110 \\
$\mathrm{RAS}^{(3)}$ & 21,1 & - \\
Classificação $^{(4)}$ & $\mathrm{C}_{4} \mathrm{~S}_{2}$ & - \\
\hline
\end{tabular}

(1) Resolução Consema 128/2006 para arroio Cascalho, Utresa, Estância Velha, RS (Consema, 2006). ${ }^{(2)} \mathrm{CE}$ : condutividade elétrica. (3) RAS: relação de adsorção de sódio, $\mathrm{RAS}=\mathrm{Na} / \sqrt{(\mathrm{Ca}+\mathrm{Mg}) / 2}$, em que $\mathrm{Na}, \mathrm{Ca}$ e Mg são utilizados em mmol L ${ }^{-1}$. ${ }^{(4)}$ Classificação para uso agrícola conforme Richards (1954). 
Coletores de solução do solo foram instalados nos vasos na profundidade de $10 \mathrm{~cm}$. Os coletores consistiram de mangueiras de PVC de $5 \mathrm{~mm}$ de diâmetro conectadas a tubos coletores de PVC de $50 \mathrm{~mm}$ de diâmetro e $40 \mathrm{~mm}$ de comprimento, recobertos nas extremidades com tela de náilon (Silva et al., 2003). Pela extremidade superior da mangueira, extraíram-se $40 \mathrm{~mL}$ de solução, utilizando-se uma seringa de $10 \mathrm{~mL}$. As coletas de solução do solo foram realizadas a partir do quarto dia após o alagamento (DAA), até 88 DAA, com intervalo de sete dias entre as coletas. A solução do solo foi coletada com seringa em um frasco isolado do contato com o ar, onde foi determinado o potencial redox com eletrodo de platina (Mettler Toledo SG2) aferido com soluções-tampão de $\mathrm{pH}$ 4,0 e 7,0 e convertida para $\mathrm{E}_{\mathrm{H}}$ pelo software do equipamento, conforme manual de operação. Logo após a coleta, no laboratório, foram determinados os valores de $\mathrm{pH}$ com eletrodo de vidro e condutor $\mathrm{Ag} / \mathrm{AgCl}$ (Digimed DM20) calibrado com solução-tampão de $\mathrm{pH}$ 4,0 e 7,0. A condutividade elétrica foi determinada com eletrodo de platina (Digimed DM31) calibrado com solução-padrão de $1.412 \mu \mathrm{S} \mathrm{cm}{ }^{-1}$. Posteriormente, os teores de $\mathrm{P}, \mathrm{K}, \mathrm{S}, \mathrm{Ca}, \mathrm{Mg}$ e Na da solução do solo foram quantificados por espectrometria de emissão atômica por plasma indutivamente acoplado (ICP), e os teores de $\mathrm{N}-\mathrm{NH}_{4}^{+}$e N-NO${ }_{3}^{-}$, por microdestilação Kjeldahl (Tedesco et al., 1995). O cálculo da razão de adsorção de sódio foi estabelecido conforme Richards (1954). Os valores de $\mathrm{DBO}_{5}$ foram determinados pelo método respirométrico 5210D (APHA, 2010).

As variáveis analisadas foram proporção de lixiviado na irrigação e no tempo de alagamento. Os resultados foram avaliados pela análise da variância a $5 \%$ de probabilidade e as médias, quando significativas, foram comparadas pelo teste de Tukey $(p<0,05)$.

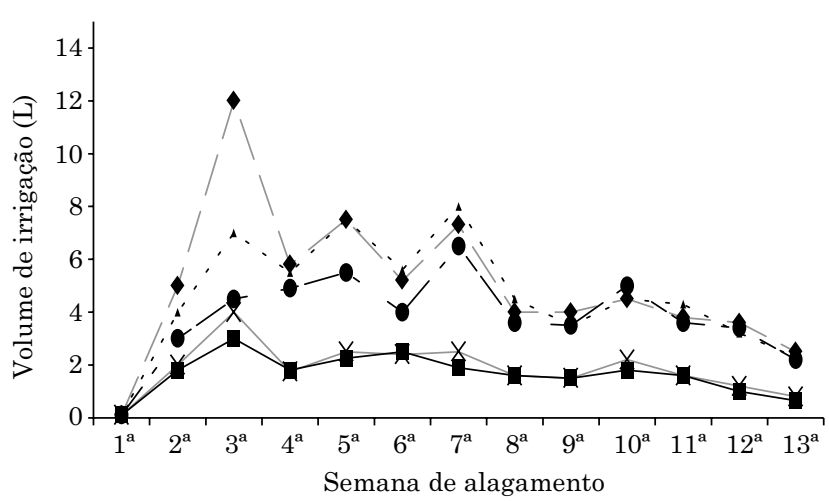

Figura 1. Volume de irrigação em 13 semanas de alagamento com diferentes proporções de

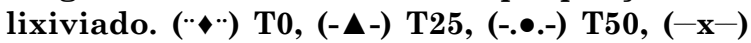
T75 e (--) T100, respectivamente 0, 25, 50, 75 e $100 \%$ de lixiviado na irrigação. Média de três repetições.

\section{RESULTADOS E DISCUSSÃO}

Airrigaçãocomolixiviadoalterou significativamente $(p<0,05)$ os indicadores eletroquímicos e a dinâmica dos nutrientes avaliados, e esses resultados estão relacionados às quantidades de carbono orgânico dissolvido (COD) e de nutrientes aportados pela irrigação (Quadro 3; Figuras 2, 3, 4 e 5). As maiores quantidades adicionadas de nutrientes foram observadas nas proporções intermediárias de lixiviado na irrigação, no T50 principalmente (Quadro 3). Possivelmente, as altas proporções de lixiviado na irrigação reduziram o crescimento vegetal, com menor evapotranspiração e, consequentemente, redução no volume de irrigação (Figura 1). O lixiviado aportou quantidades elevadas de nutrientes para o solo, muito além do considerado necessário para obter altos rendimentos da cultura (Sosbai, 2012). Os valores de COD adicionado situaram-se entre 110 a $183 \mathrm{~kg} \mathrm{ha}^{-1}$, com uma média de $153 \mathrm{~kg} \mathrm{ha}^{-1}$ e não variando muito entre os tratamentos. Observou-se que, além dos nutrientes, as quantidades de $\mathrm{Na}$ adicionadas foram consideradas tóxicas ou deletérias para a cultura (Fonseca et al., 2005; Leal et al., 2009) (Quadro 3).

Em relação aos macronutrientes, a irrigação com o lixiviado aumentou os teores de P, S, $\mathrm{N}-\mathrm{NH}_{4}^{+}$e $\mathrm{N}-\mathrm{NO}_{3}^{-}$em solução (Figura 2). O tratamento-controle (T0) apresentou teores de $\mathrm{P}$ menores que $0,3 \mathrm{mg} \mathrm{L}^{-1}$, ao passo que onde foi adicionado o lixiviado os teores de $\mathrm{P}$ situaram-se entre 0,3 e $0,8 \mathrm{mg} \mathrm{L}^{-1}$, na maioria do período de alagamento (Figura $2 \mathrm{a}$ ). $\mathrm{O}$ teor de $\mathrm{P}$ do lixiviado (Quadro 2) pode ter sido o responsável direto por esses valores, bem como indiretamente pela redução de óxidos de $\mathrm{Fe}$ e liberação de $\mathrm{P}$, decorrente do alagamento (não avaliado neste trabalho). Apesar do maior aporte de $\mathrm{P}$ pelo lixiviado, esses valores ainda são muito abaixo do que o recomendado para a cultura.

Quadro 3. Quantidades dos nutrientes, carbono orgânico dissolvido (COD) e sódio adicionados pela irrigação com o lixiviado industrial tratado (LIT) de acordo com os tratamentos e as doses dos nutrientes recomendadas para a região

\begin{tabular}{lrrrrrrrr}
\hline Trat. $^{(1)}$ & $\mathbf{N}$ & $\mathbf{P}_{2} \mathbf{O}_{5}$ & $\mathbf{K}_{\mathbf{2}} \mathbf{O}$ & $\mathbf{C a O}$ & $\mathbf{M g O}$ & $\mathbf{S}$ & $\mathbf{C O D}$ & $\mathbf{N a}$ \\
\hline & \multicolumn{8}{c}{$\mathbf{k g ~ h a}^{-1}$} \\
T25 & 330 & 3 & 300 & 78 & 117 & 32 & 110 & 1.230 \\
T50 & 547 & 5 & 498 & 129 & 194 & 52 & 183 & 2.041 \\
T75 & 465 & 5 & 422 & 110 & 165 & 44 & 165 & 1.734 \\
T100 & 398 & 4 & 316 & 94,5 & 141 & 38 & 155 & 1.485 \\
Sosbai $^{(2)}$ & 120 & 60 & 90 & - & - & - & - & -
\end{tabular}

(1) Tratamentos: 25, 50, 75 e $100 \%$ do volume da água de irrigação aplicado como LIT. ${ }^{(2)}$ Recomendações de adubação de acordo com Sociedade Sul Brasileira de Arroz Irrigado (Sosbai) para muito alta expectativas de rendimentos de grãos. 

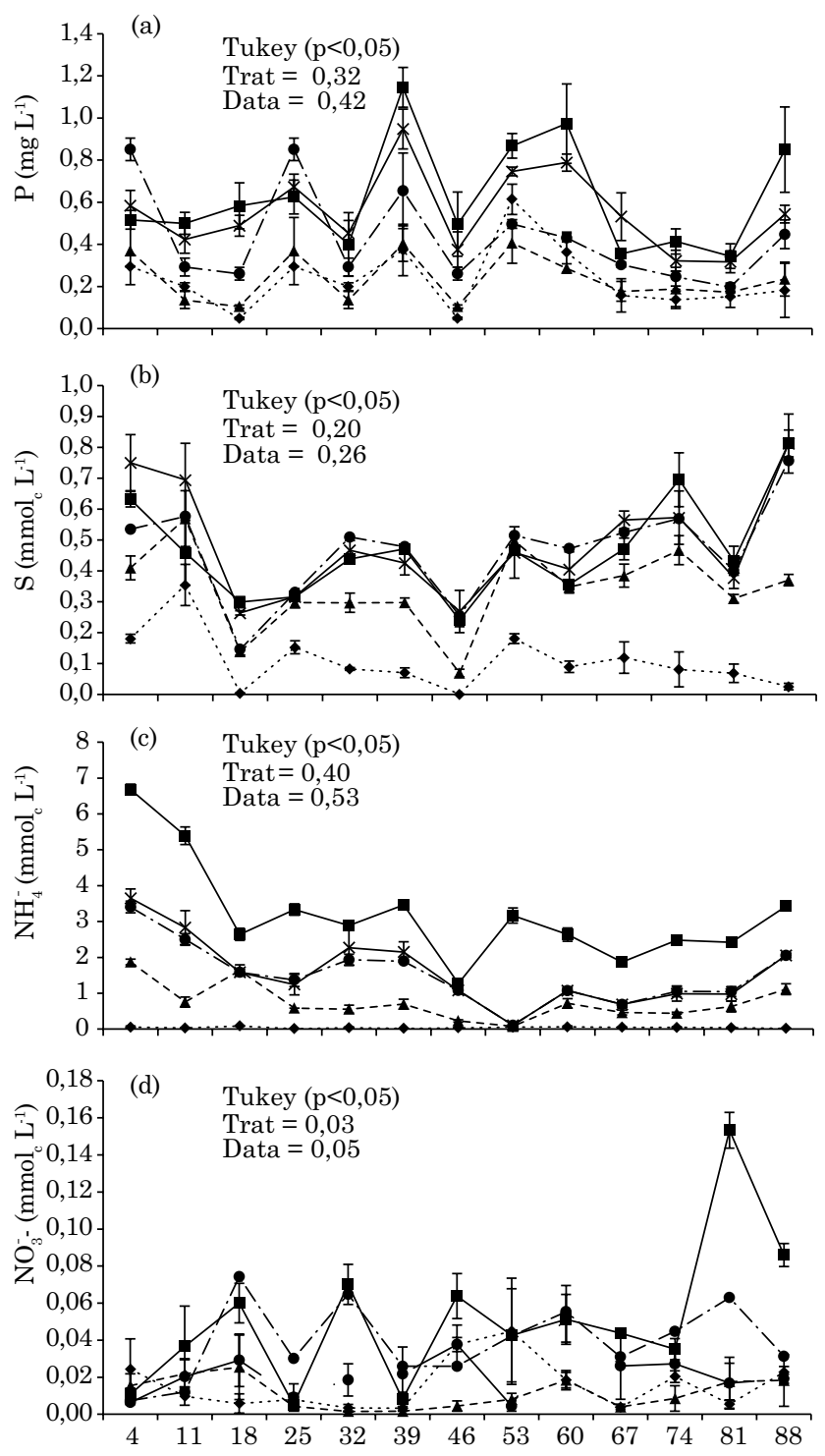

Figura 2. Teores médios de fósforo (a), enxofre (b), amônio (c) e nitrato (d) na solução do solo sob irrigação com lixiviado industrial tratado (LIT) em diferentes proporções, em 88 dias de alagamento. (*-*) T0, (-ム-) T25, (-x-) T75, (-.๑.-) T50 e (---) T100, respectivamente 0,25 , 50,75 e $100 \%$ de lixiviado na irrigação. Barras verticais indicam erro-padrão. Os valores de trat e a data nos gráficos indicam os valores de diferença mínima significativa $(\mathrm{dms})$ para os tratamentos e as datas, respectivamente, pelo teste de Tukey $(p<0,05)$.

Os teores de S na solução do solo irrigado com lixiviado também aumentaram e tiveram grande contribuição pelo lixiviado que continha $21 \mathrm{mg} \mathrm{L}^{-1} \mathrm{de}$ S. Os valores de $\mathrm{S}$ decresceram nas primeiras semanas de alagamento, estabilizando posteriormente e aumentando no final, principalmente nos tratamentos onde o lixiviado foi adicionado
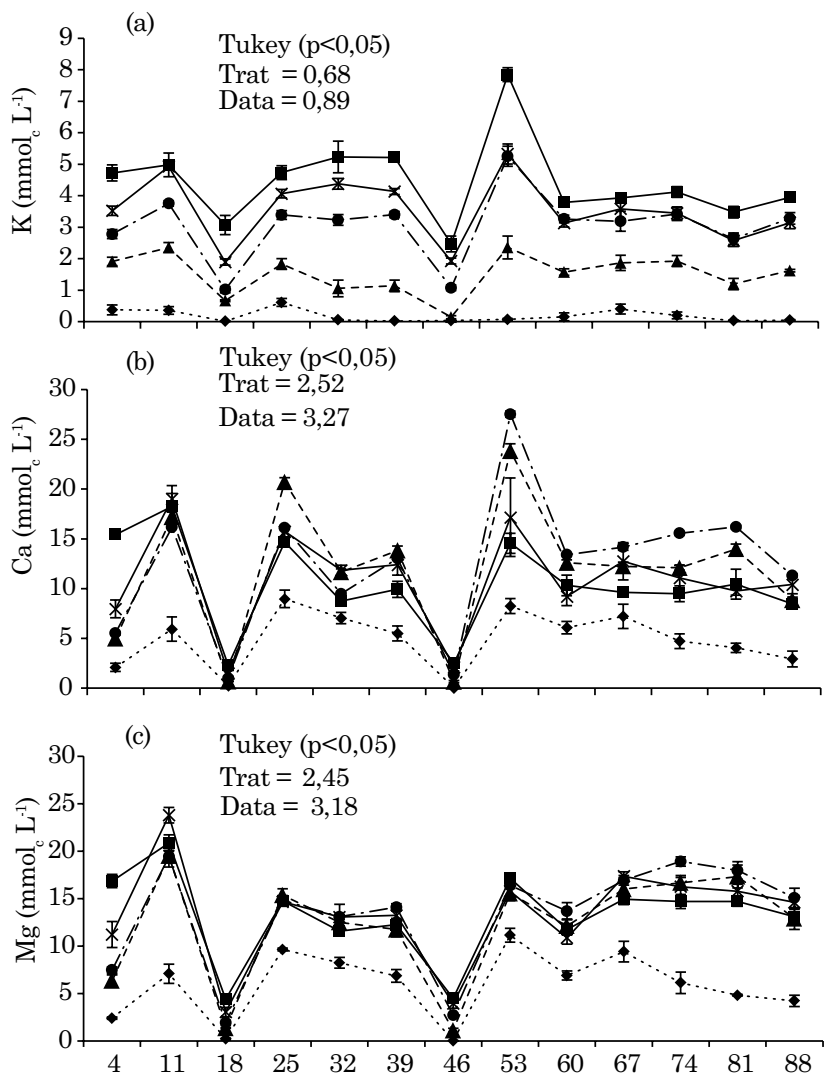

Figura 3. Teores de potássio (a), cálcio (b) e magnésio (c) na solução do solo sob irrigação com lixiviado industrial tratado (LIT) em diferentes proporções durante 88 dias de


T75 e (--) T100, respectivamente 0, 25, 50, 75 e $100 \%$ de lixiviado na irrigação. Barras verticais indicam erro-padrão. Os valores de trat e data nos gráficos indicam os valores de diferença mínima significativa (dms) para os tratamentos e datas, respectivamente, pelo teste de Tukey $(p<0,05)$.

(Figura 2b). Esse decréscimo pode estar relacionado à redução de formas oxidadas de enxofre $\left(\mathrm{SO}_{4}^{2}\right)$, que na sequência termodinâmica pode ter sido utilizado como eletroaceptor na respiração anaeróbia dos microrganismos. Na ausência de outros aceptores mais oxidados como o $\mathrm{N}-\mathrm{NO}_{3}^{-}$, a redução do $\mathrm{S}$ pode ocorrer, principalmente entre $\mathrm{pH} 5,0$ e 9,0, gerando gases como o ácido sulfídrico $\left(\mathrm{H}_{2} \mathrm{~S}\right)$. Em baixas concentrações, esse gás é altamente tóxico para plantas de arroz irrigado (Camargo et al., 1993).

Em relação aos teores de $\mathrm{N}-\mathrm{NH}_{4}^{+}$, verificaram-se os maiores valores no início do alagamento e a diminuição posterior com o alagamento (Figura 2c). Esse decréscimo pode estar relacionado à absorção pelas plantas, oxidação na rizosfera e difusão para a zona reduzida, com posterior desnitrificação para formas gasosas (Knoblauch et al., 2014). O 


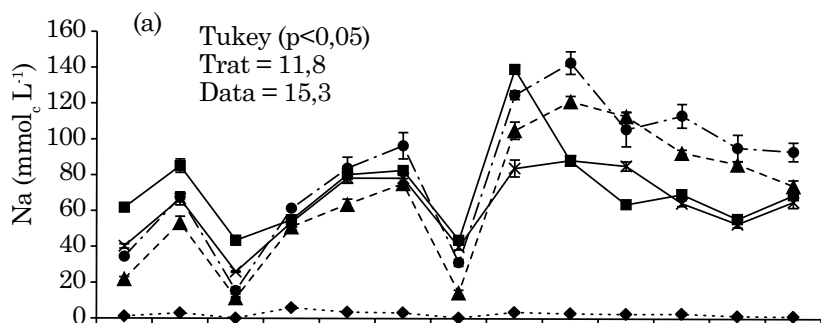

(b)

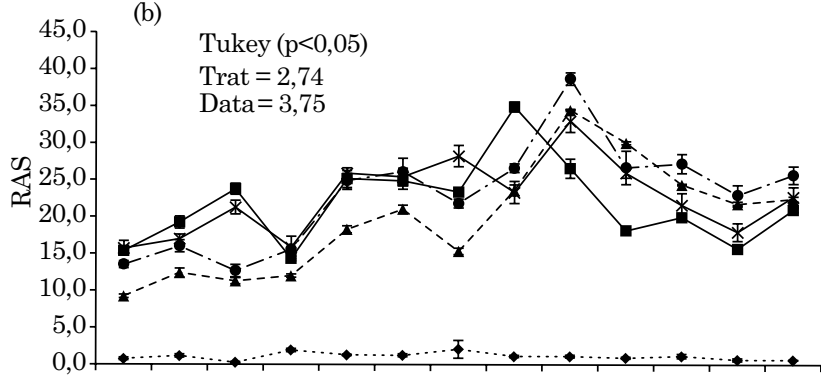

(c)

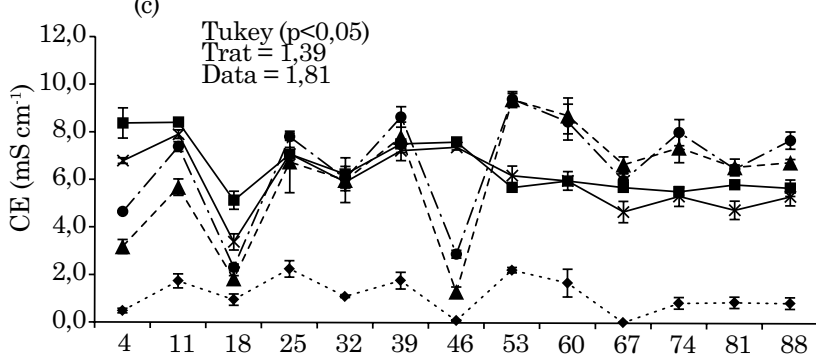

Figura 4. Valores de sódio (a), razão de adsorção de sódio (b) e condutividade elétrica (c) na solução do solo sob irrigação com lixiviado industrial tratado (LIT) em diferentes proporções durante

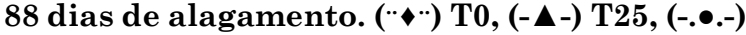
T50, $(-\mathbf{x}-)$ T75 e (--) T100, respectivamente $0,25,50,75$ e $100 \%$ de lixiviado na irrigação. Barras verticais indicam erro-padrão. Os valores de trat e data nos gráficos indicam os valores de diferença mínima significativa para os tratamentos e datas, respectivamente, pelo teste de Tukey $(p<0,05)$.

maior enraizamento das plantas na metade final do alagamento pode ter aumentado as reações de desnitrificação e também causado maior absorção de $\mathrm{N}-\mathrm{NH}_{4}^{+}$e N-NO $\mathrm{N}_{3}^{-}$pelas plantas. Além desses fatores, é possível que o menor volume de irrigação (Figura 1) no período final de alagamento também tenha contribuído para a diminuição dos teores de $\mathrm{N}-\mathrm{NH}_{4}^{+}$em solução.

Observou-se, também, pequeno aumento nos teores de $\mathrm{N}_{-} \mathrm{NO}_{3}^{-}$nos tratamentos irrigados com lixiviado (Figura 2d). A maioria dos valores observados situou-se na faixa de 0,01 a $0,08 \mathrm{mmol}_{\mathrm{c}} \mathrm{L}^{-1}$, enquanto no tratamento-controle os teores foram inferiores a $0,01 \mathrm{mmol}_{\mathrm{c}} \mathrm{L}^{-1}$. Em solos alagados, os teores de $\mathrm{N}^{-\mathrm{NO}_{3}^{-}}$são baixos (Ponnamperuma, 1972); no entanto, é provável que a disponibilidade de $\mathrm{N}-\mathrm{NH}_{4}^{+}$constante pelo lixiviado nas zonas oxidadas

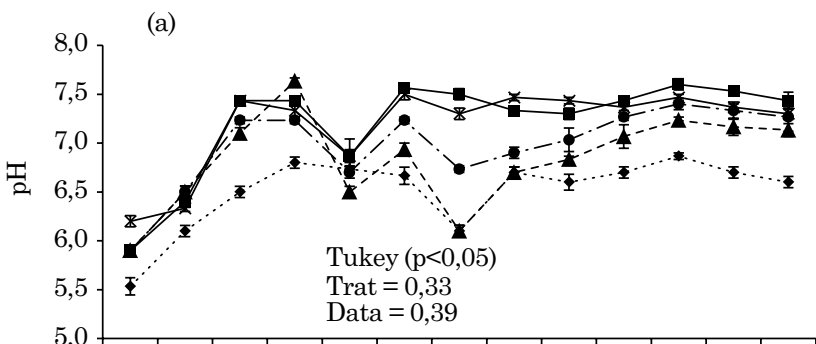

(b)

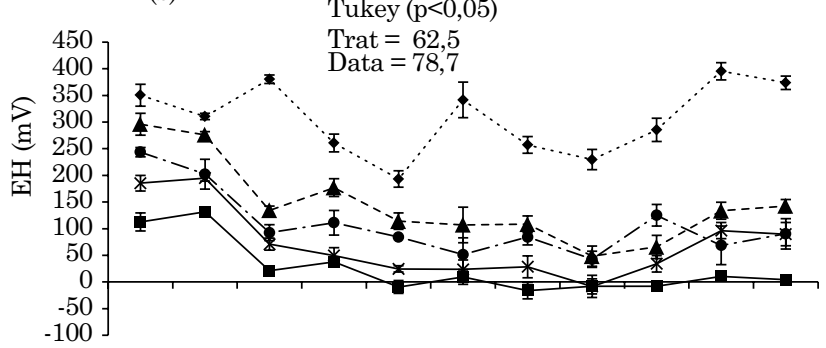

(c)

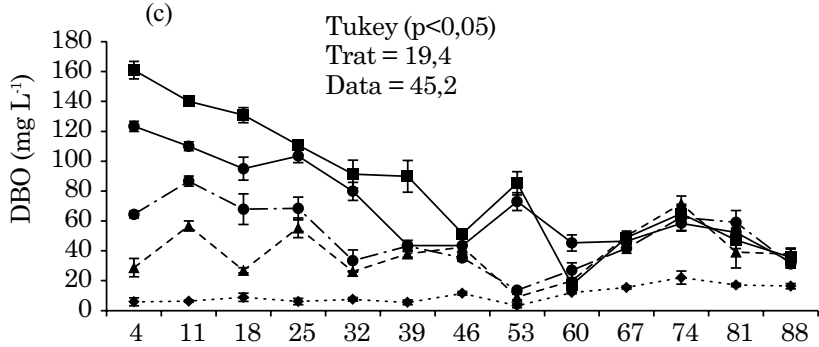

Figura 5. Valores de pH (a), $\mathrm{E}_{\mathrm{H}}(\mathrm{b})$ e $\mathrm{DBO}_{5}$ (c) na solução do solo sob irrigação com lixiviado industrial tratado (LIT) em diferentes concentrações

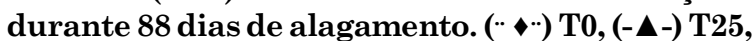
(-.๑.-) T50, (-x-) T75 e (--) T100, respectivamente 0, 25, 50, 75 e $100 \%$ de lixiviado na irrigação. Barras verticais indicam erro-padrão. Os valores de trat e data nos gráficos indicam os valores de diferença mínima significativa (dms) para os tratamentos e datas, respectivamente, pelo teste de Tukey $(p<0,05)$.

como a rizosfera e a camada superficial do solo contribuiu para a nitrificação e o aumento dos teores de $\mathrm{NO}_{3}^{-}$. Holzschuh et al. (2011) verificaram que o $\mathrm{N}-\mathrm{NO}_{3}^{-}$, embora em proporção menor que o N-NH$H_{4}^{+}$, tem função importante na absorção de $\mathrm{N}$ em arroz irrigado.

A dinâmica de nutrientes importantes como o $\mathrm{K}, \mathrm{Ca}$ e $\mathrm{Mg}$ presentes na solução do solo também foi avaliada (Figura 3), bem como o $\mathrm{Na}$, abundante no lixiviado e principal problema para a cultivo do arroz. Os teores de K na solução do solo (Figura 3a) são significativamente maiores nos tratamentos irrigados com o lixiviado e esses valores estão relacionados às grandes quantidades totais de $\mathrm{K}_{2} \mathrm{O}$ adicionadas nos tratamentos irrigados com lixiviado (Quadro 3). Aos 18 e 46 dias após o alagamento (DAA), por efeito da simulação de chuva, de $100 \mathrm{~mm}$ em cada data, verificou-se 
redução acentuada dos íons em solução em razão do efeito da diluição causada pelo incremento de água nos vasos (Figura 1). A incidência de chuva é um ponto-chave em solos que recebem disposição de águas residuárias, pois reduzem, em parte, o efeito salino, especialmente do $\mathrm{Na}$ (Fonseca et al., 2005; Leal et al., 2009). O K presente nas águas residuárias contribui para a fertilidade, especialmente de solos tropicais com baixa CTC e baixa fertilidade natural (Fonseca et al., 2005). Os aumentos dos teores de $\mathrm{Fe}^{2+}$ e $\mathrm{Mn}^{2+}$ ocasionados pela redução dos óxidos podem ter deslocado maiores quantidades de $\mathrm{K}^{+}$do complexo sortivo (Ponnampereuma, 1972). Além do teor de K do lixiviado, é possível que os altos teores de $\mathrm{Na}$ em solução adicionados pelo lixiviado tenham deslocado também parte do $\mathrm{K}^{+}$dos sítios de troca e contribuído para o aumento do K em solução (Ribeiro et al., 2009). À exceção das simulações de chuva, os teores de K em solução não apresentaram grandes oscilações nas datas de coleta, verificando-se teores na faixa de 1 a $5 \mathrm{mmol}_{\mathrm{c}} \mathrm{L}^{-1}$ nos tratamentos irrigados com lixiviado e menor que $0,5 \mathrm{mmol}_{\mathrm{c}} \mathrm{L}^{-1}$ no T0.

A irrigação com lixiviado também aumentou os teores de Ca na solução (Figura 3b) em razão das altas quantidades de $\mathrm{Ca}$ adicionados pela irrigação com lixiviado (Quadro 3). Nos tratamentos irrigados com lixiviado, os teores observados situaram-se na faixa de 8 a $20 \mathrm{mmol}_{\mathrm{c}} \mathrm{L}^{-1}$, enquanto o T0 foi de 3 a $8 \mathrm{mmol}_{\mathrm{c}} \mathrm{L}^{-1}$. A partir dos $53 \mathrm{DAA}$, os teores de Ca observados no T25 e T50 foram maiores que o T100. Sugere-se que esse efeito seja pelo maior desenvolvimento das plantas irrigadas com concentrações intermediárias de lixiviado (T25 e T50), resultando em maior transpiração e volume de irrigação (Figura 1). Os altos teores de $\mathrm{Na}$ em solução também podem deslocar o Ca dos sítios de troca (Ribeiro et al., 2009).

A irrigação com lixiviado também aumentou os teores de $\mathrm{Mg}$ em solução (Figura 3c), de forma semelhante ao Ca. Nos tratamentos com irrigação de água residuária, foram observados teores na faixa de 10 a $20 \mathrm{mmol}_{\mathrm{c}} \mathrm{L}^{-1}$, com exceção da primeira semana e das datas de simulação de chuva; e no T0, teores na faixa de 5 a $10 \mathrm{mmol}_{\mathrm{c}} \mathrm{L}^{-1}$. Além da adição do $\mathrm{Mg}$ pelo lixiviado, os altos teores de $\mathrm{Na}$ por efeito de massa podem ter contribuído para deslocar o $\mathrm{Mg}^{2+}$ do complexo de troca, bem como para os demais cátions (Ribeiro et al., 2009). Outros trabalhos também relatam os aumentos dos teores de $\mathrm{Ca}$ e $\mathrm{Mg}$ no solo a partir da irrigação com águas residuárias tratadas (Fonseca et al., 2005; Rana et al., 2010; Blum et al., 2012).

Os teores de $\mathrm{Na}$ em solução também aumentaram sob irrigação com o lixiviado (Figura 4a). Até os $18 \mathrm{DAA}$, os maiores teores foram observados no T100. Após esse período, constataram-se maiores teores de $\mathrm{Na}$ nos tratamentos com diluição intermediária (T25 e T50) em razão do maior volume de irrigação, conforme observado para $\mathrm{Ca}$ e $\mathrm{Mg}$. No início da irrigação com lixiviado, independentemente da diluição, observaram-se teores na faixa de $60 \mathrm{mmol}_{\mathrm{c}} \mathrm{L}^{-1}$. As simulações de chuva aos $18 \mathrm{e}$ 46 DAA não diminuíram o efeito salino da irrigação com o lixiviado. Assim, sob irrigação contínua, os teores de $\mathrm{Na}^{+}$observados aos 53 DAA foram próximos a $120 \mathrm{mmol}_{\mathrm{c}} \mathrm{L}^{-1}$, sendo considerados prejudiciais ao desenvolvimento de plantas (Kirk, 2001; Rana et al., 2010). Vários autores têm verificado que a adição de águas residuárias ao solo contribui para o aumento da sodicidade do solo (Fonseca et al., 2005; Rana et al., 2010; Blum et al., 2012).

A razão de adsorção de sódio (RAS) observada foi o reflexo dos altos teores de $\mathrm{Na}$ (Figura 4b). Nos tratamentos irrigados com lixiviado, as RAS, na grande maioria, foram superiores a 20 , indicando o alto risco de sodicidade da solução do solo irrigado com esse lixiviado tratado. Embora tenham sido feitas simulações de chuva aos 18 e 46 DAA, essas não foram suficientes para reduzir os teores de $\mathrm{Na}$. Os altos teores de $\mathrm{Na}$, pela irrigação com águas residuárias, podem aumentar o teor de argila dispersa, obstruir poros, dificultar a penetração radicular, diminuir a condutividade hidráulica do solo e influenciar negativamente o desenvolvimento de plantas (Coppola et al., 2004; Rana et al., 2010).

Conforme Hamilton et al. (2007), efluentes tratados industriais e domésticos possuem valores médios de RAS na faixa de 4,5 a 8 , e valores de RAS acima de 3 podem interferir negativamente as características do solo, especialmente quando a condutividade elétrica dele for menor do que $1 \mathrm{mS} \mathrm{cm}^{-1} \mathrm{e} \mathrm{o} \mathrm{pH}$ for superior à neutralidade.

Os valores de condutividade elétrica (CE) foram maiores nos tratamentos irrigados com o lixiviado (Figura 4c). Esse efeito ocorreu por causa do alto teor de sais adicionados pelo lixiviado na irrigação, principalmente o Na (Quadro 3). A partir dos $46 \mathrm{DAA}$, verificou-se aumento nos valores de CE no T25 e T50. Esse efeito deve-se à maior concentração de íons em solução como $\mathrm{K}, \mathrm{Ca}, \mathrm{Mg}$ e, principalmente, o $\mathrm{Na}$, que aumentam a condutividade elétrica. Alguns autores atribuem valores limitantes de CE para o adequado desenvolvimento de plantas de arroz (Khatun e Flowers, 1995; Carmona et al., 2011); variedades de arroz do grupo índica, cultivadas no sul do Brasil, com valores de CE maiores que $2 \mathrm{mS} \mathrm{cm}^{-1}$, têm o seu desenvolvimento comprometido, diminuição do perfilhamento, menor produção de fitomassa, aumento da esterilidade de grãos e menor rendimento de grãos (Carmona et al., 2011).

$\mathrm{O}$ pH da solução do solo foi influenciado pela irrigação com o lixiviado (Figura 5a). Os maiores valores de $\mathrm{pH}$ foram verificados nos tratamentos com lixiviado adicionado na irrigação. Observaram-se no início do alagamento valores entre 5,5 e 6,3, e após 88 dias de alagamento os valores situaram-se próximos a 7,4 para os solos irrigados com lixiviado e 6,5 no T0. Provavelmente, a grande alcalinidade 
do lixiviado e o alto teor de COD do lixiviado (Quadro 2), que acelera as reações redox, foram os responsáveis pelos aumentos do $\mathrm{pH}$. Sousa (2001), avaliando o efeito da adição de resíduos de aveia, ervilhaca e azevém em um Planossolo, verificou o maior valor de $\mathrm{pH}$ de 6,4 com 35 dias de alagamento. Dynia e Barbosa Filho (1993), avaliando a calagem em um Gleissolo, verificaram valores de $\mathrm{pH}$ na solução do solo corrigida próximos a 7,0 com oito semanas de inundação.

Os valores de potencial redox nos tratamentos irrigados com lixiviado foram próximos a $-300 \mathrm{mV}$ aos 39 DAA, enquanto em solos no T0, na mesma data, verificou-se potencial redox próximo a $-100 \mathrm{mV}$ (Figura 5b). Esses valores estão associados ao grande aporte de material orgânico pelo lixiviado, sendo a matéria orgânica, nesse caso, a principal fonte de elétrons, intensificando as reações de redução no ambiente anaeróbio (Camargo et al., 1999). Após 74 DAA, constatou-se aumento nos valores de $\mathrm{E}_{\mathrm{H}}$ provavelmente em razão do maior volume de raízes característico do início do estádio reprodutivo e, consequentemente, da maior difusão de oxigênio via aerênquima, promovendo maior oxidação do solo rizosférico (Al-Omari e Fayad, 2003).

Os valores de $\mathrm{DBO}_{5}$ nos tratamentos irrigados com LIT foram altos e proporcionais ao percentual de lixiviado na irrigação até 46 DAA (Figura 5c). Verificou-se diminuição nos teores de $\mathrm{DBO}_{5}$ ao longo dos dias de alagamento. Essa diminuição é normal, pois os microrganismos presentes na água e no solo metabolizam as moléculas orgânicas e mesmo que a irrigação com o lixiviado reponha a carga orgânica há diluição pela lâmina d'água já estabelecida com menores teores de $\mathrm{C}$ dissolvido e menor $\mathrm{DBO}_{5}$ (Silva, 2007). Além disso, após os 46 dias de alagamento, houve diminuição nos volumes de irrigação e aumento no volume de raízes por causa do maior porte das plantas, possibilitando maior difusão de oxigênio na rizosfera (Holzschuh et al., 2011) e acelerando a degradação do COD.

O COD facilmente decomponível verificado pela alta demanda bioquímica de oxigênio $\left(\mathrm{DBO}_{5}\right)$ (Quadro 2) e pela grande quantidade aportada pela irrigação com o lixiviado (Quadro 3) é um dos principais fatores que aceleraram as reações de oxirredução constatadas. A natureza da matéria orgânica, a quantidade de eletroaceptores $\left(\mathrm{NO}_{3}^{-}\right.$, $\mathrm{Mn}^{4+}, \mathrm{Fe}^{3+}, \mathrm{SO}_{4}^{-}$), a temperatura e o tempo de alagamento influenciam as reações redox do solo (Camargo et al., 1999). As altas temperaturas no período de condução do experimento, o baixo teor de óxidos de $\mathrm{Fe}$ e de $\mathrm{Mn}$ no solo, o período de alagamento de 88 dias e a alta $\mathrm{DBO}_{5}$ contribuíram para os valores extremos do potencial redox nos tratamentos irrigados com o lixiviado.

Os altos teores de N-NH$+\mathrm{N}_{4}^{+}, \mathrm{NO}_{3}^{-}$e $\mathrm{DBO}_{5}$ das águas residuárias melhoram a fertilidade dos solos e a nutrição de plantas (Fonseca et al., 2007). No entanto, por causa dos altos desequilíbrios, como a eutrofização de corpos hídricos e a fragilidade ambiental (proximidade do lençol freático e corpos hídricos) dos solos de cultivo de arroz, é necessário monitoramento desses compostos. Poucos trabalhos abordam a aplicação de efluentes tratados em arroz irrigado em que avaliam a dinâmica da $\mathrm{DBO}_{5}$ e de formas minerais de $\mathrm{N}$ na solução do solo.

Baixos volumes de lixiviado são recomendados na irrigação por alagamento do arroz que é uma cultura moderadamente sensível ao Na. Proporções inferiores a $25 \%$ devem ser utilizadas para reduzir os níveis de sodicidade, além de diminuir a concentração de $\mathrm{NH}_{4}^{+}$e os valores de $\mathrm{DBO}_{5}$, que são potenciais contaminantes. A calagem e a gessagem são práticas importantes nas áreas de aplicação de lixiviado industrial tratado, pois a adição de íons divalentes como $\mathrm{Ca}$ e $\mathrm{Mg}$ por esses insumos minimiza os efeitos negativos da sodicidade, e o volume de lixiviado a ser aplicado deve ser limitado com base no seu teor de $\mathrm{Na}$, mesmo que o $\mathrm{Na}$ não altere o desenvolvimento vegetal; em alguns casos, pode aumentar a dispersão de argila, obstruir poros e influenciar negativamente os componentes físicos do solo (Coppola et al., 2004; Rana et al., 2010).

\section{CONCLUSÕES}

Os teores de potássio, cálcio, magnésio, fósforo, amônio, nitrato e enxofre aumentam na solução do solo sob irrigação com lixiviado industrial tratado.

A irrigação com lixiviado industrial tratado aumenta os teores de sódio, assim como valores da RAS e da condutividade elétrica na solução do solo em níveis considerados prejudiciais para o desenvolvimento de plantas.

O potencial redox da solução do solo decresce para potenciais negativos, e os valores de $\mathrm{pH}$ aumentam quando irrigado com lixiviado industrial tratado.

A demanda bioquímica de oxigênio da solução do solo aumenta sob irrigação com lixiviado industrial tratado e diminui com o tempo de alagamento, assim como o amônio.

As proporções inferiores a $25 \%$ de lixiviado são recomendadas na irrigação de arroz, pois as concentrações de potenciais contaminantes como $\mathrm{DBO}_{5}$ e $\mathrm{NH}_{4}^{+}$e $\mathrm{Na}^{+}$não atingem valores críticos e ainda aumentam os teores de nutrientes em solução.

\section{REFERÊNCIAS}

Al-Omari A, Fayyad M. Treatment of domestic wastewater by subsurface flow constructed wetlands in Jordan. Desalination. 2003;155:27-39. 
American Public Health Association - APHA. Standard methods for the examination of water and wastewater. $181^{\mathrm{a}} \mathrm{ed}$. Washington; 2005.

Blum J, Herpin U, Melfi AJ, Montes CR. Soil properties in a sugarcane plantation after the application of treated sewage effluent and phosphogypsum in Brazil. Agric Water Manage. 2012;115:203-16.

Camargo FAO, Santos GA, Rossiello ROP. Produção de ácidos orgânicos voláteis pela planta de arroz sob condições anaeróbias. R Bras Ci Solo. 1993;17:337-42.

Camargo FAO, Santos GA, Zonta E. Alterações eletroquímicas em solos inundados. Ci Rural. 1999;29:171-80.

Carmona FC, Anghinoni I, Holzschuh MJ, Martins AP. Attributes of irrigated rice as affected by soil sodicity and potassic fertilizer application. R Bras Ci Solo, 2011;35:889-97.

Comissão de Química e Fertilidade do Solo - CQFSRS/SC. Manual de adubação e de calagem para os estados do Rio Grande do Sul e de Santa Catarina. 10 a ed. Porto Alegre: Sociedade Brasileira de Ciência do Solo; 2004.

Coppola A, Santini A, Botti P, Vacca S, Comegna V, Severino G. Methodological approach for evaluating the response of soil hydrological behavior to irrigation with treated municipal wastewater. J Hydrol, 2004;292:114-34.

Conselho Estadual do Meio Ambiente - Consema. Lei Estadual $\mathrm{n}^{\circ} 10.330$, de 27 de dezembro de 1994. Secretaria do Meio Ambiente do Estado do Rio Grande do Sul. Resolução Consema $\mathrm{n}^{\circ} 128$ de 2006

Dynia JF, Barbosa Filho MP. Alterações de pH, Eh e disponibilidade de micronutrientes para arroz irrigado em um solo de várzea tratado com calcário e palha de arroz em casa de vegetação. R Bras Ci Solo. 1993;17:67-74.

Empresa Brasileira de Pesquisa Agropecuária - Embrapa. Sistema brasileiro de classificação de solos. $2^{\mathrm{a}}$ ed. Rio de Janeiro: Centro Nacional de Pesquisa de Solos; 2006.

Feigin A, Ravina I, Shalhevet J. Irrigation with treated sewage effluent: Management for environmental protection. Berlin: Springer-Verlag; 1991.

Fonseca AF, Melfi AJ, Montes CR. Maize growth and changes in soil fertility after irrigation with treated sewage effluent in soil acidity, exchangeable cations, and sulfur, boron and heavy metals availability. Soil Sci Plant Anal. 2005;36:1983-2003.

Fonseca AF, Herpin U, Paula AM, Victoria RL, Melfi AJ. Agricultural use of treated sewage effluents: Agronomical environmental implications and perspectives for Brazil. Sci Agric. 2007;64:194-209.

Hamilton AJ, Stagnitti F, Xiong X, Kreidl SL, Benke KK, Maher, P. Wastewater irrigation: The state of play. Vad Zone J. 2007;6:823-40.

Holzschuh MJ, Bohnen H, Anghinoni I, Pizzolato TM, Carmona FC, Carlos FS. Absorção de nutrientes e crescimento do arroz com suprimento combinado de amônio e nitrato. $\mathrm{R}$ Bras Ci Solo. 2011;35:1357-66.
Instituto Brasileiro de Geografia e Estatística - IBGE. Indicador de desenvolvimento sustentável. Rio de Janeiro; 2010. (Estudos e pesquisas - Informações geográficas, 7).

Khatun S, Flowers TJ. Effects of salinity on seed set in rice. Plant Cell Environ. 1995;18:61-7.

Kirk GJD. Plant-mediated processes to acquire nutrients: Nitrogen uptake by rice plants. Plant Soil. 2001;232:129-34.

Knoblauch R, Ernani PR, Deschamps FC, Gatiboni LC, Walker TW, Lourenço KS, Martins AA, Pegoraro A. Rice straw incorporated just before soil flooding increases acetic acid formation and decreases available nitrogen. R Bras Ci Solo. $2014 ; 38,1: 177-84$.

Leal RMP, Herpin U, Fonseca AF, Firme LP, Montes CR, Melfi AJ. Sodicity and salinity in a Brazilian Oxisol cultivated with sugarcane irrigated with wastewater. Agric Water Manage. 2009;96:307-16.

Lourenzi CR, Ceretta CA, Silva LS, Girotto E, Lorensini F, Tiecher, TL, De Conti, L, Trentin G, Brunetto G. Nutrients in soil layers under no-tillage after successive pig slurry applications. R Bras Ci Solo. 2013;37,1:157-67.

Ponnamperuma FN. The chemical of submerged soils. Adv Agron. 1972;24:29-96.

Rana L, Dhankhar R, Chhikara S. Soil characteristics affected by long-term application of sewage wastewater. Inter J Environ Res. 2010;4:513-8.

Ribeiro MR, Barros MFC, Freire MBGS. Química dos solos salinos e sódicos. In: Melo VF, Alleoni LR, editores. Química e mineralogia do solo. Viçosa, MG: Sociedade Brasileira de Ciência do Solo; 2009. v.2, p.449-84.

Richards LA. Diagnosis and improvement of saline and alkali soils. Washington: United States Department of Agriculture; 1954. (Agriculture handbook, 60).

Silva SC. Wetlands construídos de fluxo vertical com meio suporte de solo natural modificado no tratamento de esgotos domésticos [tese]. Brasília: Universidade de Brasília; 2007.

Silva LS, Sousa RO, Bohnen, H. Alterações nos teores de nutrientes em dois solos alagados, com e sem plantas de arroz. Ci Rural, 2003;33:487-90.

Sociedade Sul-Brasileira de Arroz Irrigado - Sosbai. Arroz irrigado: recomendações técnicas da pesquisa para o sul do Brasil. In: Anais da Reunião Técnica Sul-Brasileira de Arroz, 2012; Gravatal. Gravatal; 2012.

Sousa RO. Oxirredução em solos alagados afetada por resíduos vegetais [tese]. Porto Alegre: Universidade Federal do Rio Grande do Sul; 2001.

Sousa RO, Bohnen H, Meurer EJ. Composição da solução de um solo alagado conforme a profundidade e o tempo de alagamento, utilizando novo método de coleta. R Bras Ci Solo. 2002;26:343-8.

Tedesco MJ, Gianello C, Bissani CA, Bohnen H, Volkweiss SJ. Análise de solo, plantas e outros materiais. Porto Alegre: Universidade Federal do Rio Grande do Sul; 1995. (Boletim técnico, 5). 